HEPHY-PUB 964/16

March 2016

\title{
INSTANTANEOUS BETHE-SALPETER KERNEL FOR THE LIGHTEST PSEUDOSCALAR MESONS
}

\author{
Wolfgang LUCHA* \\ Institute for High Energy Physics, \\ Austrian Academy of Sciences, \\ Nikolsdorfergasse 18, A-1050 Vienna, Austria \\ Franz F. SCHÖBERL ${ }^{\dagger}$ \\ Faculty of Physics, University of Vienna, \\ Boltzmanngasse 5, A-1090 Vienna, Austria
}

\begin{abstract}
Starting from a phenomenologically successful, numerical solution of the Dyson-Schwinger equation that governs the quark propagator, we reconstruct in detail the interaction kernel that has to enter the instantaneous approximation to the Bethe-Salpeter equation to allow us to describe the lightest pseudoscalar mesons as quark-antiquark bound states exhibiting the (almost) masslessness necessary for them to be interpretable as the (pseudo) Goldstone bosons related to the spontaneous chiral symmetry breaking of quantum chromodynamics.
\end{abstract}

PACS numbers: 11.10.St, 03.65.Ge, 03.65.Pm

* E-mail address: wolfgang.lucha@oeaw.ac.at

† E-mail address: franz.schoeberl@univie.ac.at 


\section{Introduction}

As part of the generally accepted basis (for purely historical reasons frequently still dubbed as standard "model") of theoretical elementary particle physics, quantum chromodynamics (QCD), the relativistic quantum field theory that describes all strong interactions between its colour-carrying fundamental degrees of freedom - that is, the quarks and the gluons is (strongly believed to be) characterized by two essential properties: colour confinement expressing the empirically established fact of non-observation of isolated coloured particles in nature - and dynamical breakdown of chiral symmetry - manifested by the emergence of nonvanishing masses of nonperturbative origin for, at Lagrangian level, massless quarks.

In principle, relativistic quantum field theory describes bound states of its fundamental degrees of freedom by Bethe-Salpeter amplitudes obtained by solution of the homogeneous Bethe-Salpeter equation [1]. For technical or practical reasons, the latter is frequently used in some instantaneous limit [2], which - upon assuming, for each bound-state constituent, free propagation with constant effective mass - finally collapses to Salpeter's equation [3].

QCD enables to regard light pseudoscalar mesons as bound states of quarks and gluons, or as pseudo-Goldstone bosons whose presence is demanded by the Goldstone theorem as a consequence of spontaneous chiral symmetry breaking. Recently, we embarked on the quest for reconciling both views by a well-tailored instantaneous Bethe-Salpeter formalism [4-6].

Instead of attempting to solve a given bound-state equation in use straightforwardly, its exact solutions may be likewise determined, along a more indirect route and only with a bit of luck, by application of sufficiently sophisticated inversion techniques which establish the rigorous relation between the relevant interactions and the wave functions (in our case, the Bethe-Salpeter amplitudes) that characterize the features of the bound states under study. In Ref. [4], we demonstrated the feasibility of this inversion concept for the easier-to-handle example of the projection of the Salpeter equation to positive energies of the bound quarks.

All symmetries of a quantum field theory are reflected by its Ward-Takahashi identities rephrasing the implications of such symmetries at the level of the theory's Green functions. In Ref. [5], we employed an ensued relation between flavour-nonsinglet pseudoscalar-meson Bethe-Salpeter amplitude and quark propagator to implement the proper ultraviolet limit.

General insights, rooting in axiomatic foundations of quantum field theory and encoded in the axiom of reflection positivity, impose constraints on the propagators of those degrees of freedom of any quantum field theory that are bound to be subject to colour confinement. In Ref. [6], we added constraints on the momentum dependence of the quark mass function, implied by the nonexistence of a Källén-Lehmann representation for the quark propagator.

Within our instantaneous Bethe-Salpeter approach, the effective interaction governing bound states is fixed by the quark couplings' Lorentz nature and a central potential $V(|\boldsymbol{x}|)$. In this study, we pin down $V(|\boldsymbol{x}|)$ from a numerical solution of the quark Dyson-Schwinger equation exploiting the at present presumably most popular model for the necessary input.

The outline of this paper is as follows. In Sec. 2, we sketch, only to the extent absolutely necessary for any self-contained presentation, the established description of bound states of fermion and antifermion by means of the Bethe-Salpeter framework in instantaneous limit. In Sec. 3, we briefly recall the interplay between Bethe-Salpeter amplitude and quark mass function, established by the axial-vector Ward-Takahashi identity for QCD by relating the solution of the Bethe-Salpeter equation and that of the quark's Dyson-Schwinger equation. In Sec. 4, we recall our favourite model for the latter. In Sec. 5, we infer $V(|\boldsymbol{x}|)$ as a function of the (at this stage) only free parameter, the effective quark mass. In Sec. 6, we discuss our observations. (For convenience of notation, we adopt natural units throughout: $\hbar=c=1$.) 


\section{Mesons by Instantaneous Bethe-Salpeter Equation}

In quantum field theory, the Bethe-Salpeter approach describes a two-particle bound state in terms of its Bethe-Salpeter amplitude $\Phi(p, P)$, depending on both relative momentum $p$ and total momentum $P$ of its bound-state constituents (suppressing indices for simplicity). The homogeneous Bethe-Salpeter equation [1] controlling $\Phi(p, P)$ reduces, for bound-state constituents experiencing exclusively instantaneous interactions and having propagators of a sufficiently trivial dependence on $p_{0}$, to the instantaneous Bethe-Salpeter equation [2] for

$$
\phi(\boldsymbol{p}) \equiv \frac{1}{2 \pi} \int \mathrm{d} p_{0} \Phi(p),
$$

the (equal-time) Salpeter amplitude. If the latter requirement is satisfied by approximating the propagators by their free forms involving effective masses, the bound-state equation for $\phi(\boldsymbol{p})$ simplifies further to the Salpeter equation [3]. For bound states composed of a fermion and an antifermion (with masses $m_{1,2}$ and momenta $\boldsymbol{p}_{1,2}$, respectively), that equation reads

$$
\begin{aligned}
\phi(\boldsymbol{p})=\int \frac{\mathrm{d}^{3} q}{(2 \pi)^{3}} & \left(\frac{\Lambda_{1}^{+}\left(\boldsymbol{p}_{1}\right) \gamma_{0}[K(\boldsymbol{p}, \boldsymbol{q}) \phi(\boldsymbol{q})] \gamma_{0} \Lambda_{2}^{-}\left(\boldsymbol{p}_{2}\right)}{P_{0}-E_{1}\left(\boldsymbol{p}_{1}\right)-E_{2}\left(\boldsymbol{p}_{2}\right)}\right. \\
& \left.-\frac{\Lambda_{1}^{-}\left(\boldsymbol{p}_{1}\right) \gamma_{0}[K(\boldsymbol{p}, \boldsymbol{q}) \phi(\boldsymbol{q})] \gamma_{0} \Lambda_{2}^{+}\left(\boldsymbol{p}_{2}\right)}{P_{0}+E_{1}\left(\boldsymbol{p}_{1}\right)+E_{2}\left(\boldsymbol{p}_{2}\right)}\right),
\end{aligned}
$$

with free energy and positive/negative energy projectors of the two constituents defined by

$$
E_{i}(\boldsymbol{p}) \equiv \sqrt{\boldsymbol{p}^{2}+m_{i}^{2}}, \quad \Lambda_{i}^{ \pm}(\boldsymbol{p}) \equiv \frac{E_{i}(\boldsymbol{p}) \pm \gamma_{0}\left(\boldsymbol{\gamma} \cdot \boldsymbol{p}+m_{i}\right)}{2 E_{i}(\boldsymbol{p})}, \quad i=1,2 .
$$

Its instantaneous Bethe-Salpeter kernel $K(\boldsymbol{p}, \boldsymbol{q})$ subsumes Lorentz nature and momentum dependence of the effective interactions taking place between the bound-state constituents: the former by a set of (generalized) Dirac matrices $\Gamma_{i}(i=1,2)$, the latter by corresponding Lorentz-scalar potential functions $V_{\Gamma}(\boldsymbol{p}, \boldsymbol{q})$. For identical Lorentz structures of the effective couplings of fermion and antifermion, i.e., $\Gamma_{1}=\Gamma_{2}=\Gamma$, the action of $K(\boldsymbol{p}, \boldsymbol{q})$ on $\phi(\boldsymbol{p})$ reads

$$
[K(\boldsymbol{p}, \boldsymbol{q}) \phi(\boldsymbol{q})]=\sum_{\Gamma} V_{\Gamma}(\boldsymbol{p}, \boldsymbol{q}) \Gamma \phi(\boldsymbol{q}) \Gamma .
$$

Following Refs. [7-9], we rely on the postulate of Fierz symmetry of $K(\boldsymbol{p}, \boldsymbol{q})$ by choosing as its sole Lorentz structure (whence $V(\boldsymbol{p}, \boldsymbol{q})$ no longer needs an index) the linear combination

$$
\Gamma \otimes \Gamma=\frac{1}{2}\left(\gamma_{\mu} \otimes \gamma^{\mu}+\gamma_{5} \otimes \gamma_{5}-1 \otimes 1\right)
$$

The $\Lambda^{+} \otimes \Lambda^{-}+\Lambda^{-} \otimes \Lambda^{+}$projector structure of the right-hand side of Eq. (2) implies that, in a Dirac-space basis of 16 complex $4 \times 4$ matrices, the most general solution $\phi(\boldsymbol{p})$ has eight independent scalar component functions. Out of these, ignoring flavour violation by letting $m_{1}=m_{2}=m$, just two, henceforth called $\varphi_{i}(\boldsymbol{p}), i=1,2$, correspond to spin-singlet bound states of two spin- $\frac{1}{2}$ (anti-)fermions, such as pseudoscalar mesons [10]. For these, $\phi(\boldsymbol{p})$ reads

$$
\phi(\boldsymbol{p})=\left[\varphi_{1}(\boldsymbol{p}) \frac{\gamma_{0}(\boldsymbol{\gamma} \cdot \boldsymbol{p}+m)}{E(\boldsymbol{p})}+\varphi_{2}(\boldsymbol{p})\right] \gamma_{5} .
$$

For spherically symmetric convolution-type kernels $K(\boldsymbol{p}, \boldsymbol{q})$, i.e., $V(\boldsymbol{p}, \boldsymbol{q})=V\left((\boldsymbol{p}-\boldsymbol{q})^{2}\right)$, the Salpeter equation (2) simplifies to a set of coupled equations for the radial factors $\varphi_{i}(p)$, $i=1,2, \ldots$, of the independent Salpeter components. Therein, all interactions are encoded in form of configuration-space central potentials, here generically denoted by $V(r), r \equiv|\boldsymbol{x}|$. 
Under all these assumptions, the Salpeter equation (2) can be shown to be equivalent to a system of two radial equations [8], an integral equation and a relation of algebraic nature, determining mass eigenvalue $\widehat{M}$ and radial Salpeter components $\varphi_{1,2}(p)$ of the bound state:

$$
\begin{aligned}
& 2 E(p) \varphi_{2}(p)+2 \int_{0}^{\infty} \frac{\mathrm{d} q q^{2}}{(2 \pi)^{2}} V(p, q) \varphi_{2}(q)=\widehat{M} \varphi_{1}(p), \\
& 2 E(p) \varphi_{1}(p)=\widehat{M} \varphi_{2}(p), \quad E(p) \equiv \sqrt{p^{2}+m^{2}}, \quad p \equiv|\boldsymbol{p}| .
\end{aligned}
$$

The potential $V(r)$ enters the first of these relations in form of its Fourier-Bessel transform

$$
V(p, q) \equiv \frac{8 \pi}{p q} \int_{0}^{\infty} \mathrm{d} r \sin (p r) \sin (q r) V(r), \quad q \equiv|\boldsymbol{q}|
$$

Within the context of the present kind of problem, inversion simply means to determine the underlying interaction entering into the generic type of equation of motion under study by postulating one's favoured set of solutions. We take into account the Goldstone nature of the pseudoscalar bound states to be described by Eq. (4) by requiring their mass eigenvalue $\widehat{M}$ to vanish: $\widehat{M}=0$. At this point in our space of solutions, the two dynamical equations in the set (4) decouple. The second relation implies $\varphi_{1}(p) \equiv 0$, whence the Salpeter amplitude (3) simplifies to $\phi(\boldsymbol{p})=\varphi_{2}(\boldsymbol{p}) \gamma_{5}$, and the first equation provides the sole component $\varphi_{2}(\boldsymbol{p})$ :

$$
E(p) \varphi_{2}(p)+\int_{0}^{\infty} \frac{\mathrm{d} q q^{2}}{(2 \pi)^{2}} V(p, q) \varphi_{2}(q)=0 .
$$

With the Fourier-Bessel transforms of the amplitude $\varphi_{2}(p)$ and the kinetic term $E(p) \varphi_{2}(p)$

$$
\varphi(r) \equiv \sqrt{\frac{2}{\pi}} \frac{1}{r} \int_{0}^{\infty} \mathrm{d} p p \sin (p r) \varphi_{2}(p), \quad T(r) \equiv \sqrt{\frac{2}{\pi}} \frac{1}{r} \int_{0}^{\infty} \mathrm{d} p p \sin (p r) E(p) \varphi_{2}(p),
$$

transforming Eq. (5) to configuration space enables the straightforward extraction of $V(r)$ :

$$
T(r)+V(r) \varphi(r)=0 \quad \Longleftrightarrow \quad V(r)=-T(r) / \varphi(r) .
$$

In this representation, it becomes plain that Eq. (5) is just a spinless Salpeter equation [11].

\section{The Dyson-Schwinger-Bethe-Salpeter Conspiracy}

As an immediate consequence of the (global or local) symmetries of a quantum field theory, Ward-Takahashi identities relate differing $n$-point functions, e.g., propagators and vertices. In the chiral limit, the renormalized axial-vector QCD Ward-Takahashi identity relates the dressed quark propagator $S(p)$ to the Bethe-Salpeter solution $\Phi(p, 0)$ for flavour-nonsinglet pseudoscalar mesons: among others, a propagator function basically determining the quark mass function in the former to the dominant "Dirac" component of the latter $[12,13]$. More precisely, if the two Lorentz-scalar functions characterizing the propagator of the quark are chosen to be its mass function, $M\left(p^{2}\right)$, and its wave-function renormalization factor, $Z\left(p^{2}\right)$,

$$
S(p)=\frac{\mathrm{i} Z\left(p^{2}\right)}{\not p-M\left(p^{2}\right)+\mathrm{i} \varepsilon}, \quad \not p \equiv p^{\mu} \gamma_{\mu}, \quad \varepsilon \downarrow 0,
$$

and if the - comparatively low - influence of the latter function is neglected, in the chiral limit this Bethe-Salpeter amplitude $\Phi(\underline{k}, 0)$ is found [5] to be related to $M\left(\underline{k}^{2}\right)$ according to

$$
\Phi(\underline{k}, 0) \propto \frac{M\left(\underline{k}^{2}\right)}{\underline{k}^{2}+M^{2}\left(\underline{k}^{2}\right)} \gamma_{5}+\text { subleading contributions ; }
$$

here and below, vectors in Euclidean space, where Eq. (7) has been derived, are underlined. 


\section{Quark Propagator via Dyson-Schwinger Equations}

By virtue of the relationship established by Eq. (7), the (approximate) pointwise behaviour of the momentum-space Bethe-Salpeter amplitude for the massless pseudoscalar mesons in the center-of-momentum frame can be extracted from the quark mass function entering the quark propagator. This two-point Green function, in turn, can be derived as solution of the Dyson-Schwinger equation for the quark propagator (sometimes dubbed as gap equation).

With sufficient information about the Bethe-Salpeter amplitude for the light mesons at our disposal, it is a (more or less) straightforward enterprise to chart the sought interaction potential: The Salpeter amplitude of any mesonic bound states under consideration follows by application of its definition (1). Then, the underlying potential may be read off from the configuration-space representation of the effective equation of motion according to Eq. (6).

Now, the Dyson-Schwinger equations constitute a countable infinity of coupled integral equations for the infinite set of $n$-point Green functions of a quantum field theory. However, every member of this hierarchy of relations connects $n$-point Green functions of different $n$ : each Dyson-Schwinger equation requires as input the solution of, at least, one higher-order Dyson-Schwinger equation. Thus, the definition of a tractable problem is only possible by a truncation of this infinite tower, to a finite set of equations for the Green functions of low $n$, with any required higher- $n$ input Green function modelled by phenomenological reasoning.

For obvious reasons, one's truncation of choice should best respect all the symmetries of the quantum field theory expressed by its Ward-Takahashi identities: The "rainbow-ladder truncation" is defined by a tree-level quark-gluon vertex, a Bethe-Salpeter kernel in ladder approximation relying on single-gluon exchange, and a free gluon propagator. Nonetheless, the desired conformity of the resulting predictions with constraints imposed by experiment is, on the other hand, ensured by effective coupling functions which substitute the square of the strong couplings and have been composed such as to incorporate any necessary feature. This truncation preserves, at least, the crucial QCD axial-vector Ward-Takahashi identity.

For the purposes of our present investigation, we will take advantage of the findings of a study [13] adopting a renormalization-group-improved truncation model devised such that the effective coupling strength that defines a particular rainbow-ladder truncation exhibits two decisive features: a distinctive enhancement in the infrared region $\left(\underline{k}^{2} \rightarrow 0\right)$, motivated by the apparent behaviour of the gluon propagator, and a decay similar to the perturbative behaviour of the strong fine-structure coupling of QCD in the ultraviolet region $\left(\underline{k}^{2} \rightarrow \infty\right)$.

Recognizing the importance of taking into account — in the course of application of our relation (7) - the behaviour of the quark mass function in the limit $\underline{k}^{2} \rightarrow \infty$ as correctly as possible [5], we should capture a $\underline{k}^{2}$ interval which extends to $\underline{k}^{2}$ values as large as available. Hence, we utilize the quark mass function $M\left(\underline{k}^{2}\right)$ in the form presented in Fig. 2 of Ref. [13].

\section{$5 \quad$ Interaction Potentials from Quark Mass Functions}

After these preliminaries, the potential $V(r)$ enabling the Salpeter equation (2) to describe massless pseudoscalar solutions can be derived by an unspectacular sequence of operations:

1. In order to get a firm grip on our inversion problem under consideration, we first need to convert the input information, provided in pointwise shape by Fig. 2 of Ref. [13], to a mathematical expression. We may achieve this goal by construction of a convenient parametrization of the quark mass function $M\left(\underline{k}^{2}\right)$ in terms of a very small number of elementary (and hence easy-to-handle) functions. We employ a six-parameter ansatz: 
Table 1: Numerical values of the six parameters $a, b, \gamma, \delta, c, d$ defining our formal modelling (8) of the quark mass function $M\left(\underline{k}^{2}\right)$ in the chiral limit as retrieved from Fig. 2 of Ref. [13].

\begin{tabular}{lcccccc}
\hline \hline Parameter & $a[\mathrm{GeV}]$ & $b\left[\mathrm{GeV}^{2}\right]$ & $\gamma$ & $\delta$ & $c[\mathrm{GeV}]$ & $d\left[\mathrm{GeV}^{-2}\right]$ \\
\hline Value & 0.0705896 & 0.486542 & 1.48 & 0.75188 & 0.707983 & 1.52616 \\
\hline \hline$\left(\underline{k}^{2}\right)=\frac{a}{\left[1+\left(\underline{k}^{2} / b\right)^{\gamma}\right]^{\delta}}+c \exp \left(-d \underline{k}^{2}\right)$.
\end{tabular}

Fitting our parametrization (8) of the quark mass function, $M\left(\underline{k}^{2}\right)$, to the graph of its momentum dependence in the chiral limit given in Ref. [13] provides, for the involved six parameters, $a, b, \gamma, \delta, c$, and $d$, the numerical values listed in Table 1 . Accordingly, for lightlike momenta $\left(\underline{k}^{2}=0\right), M\left(\underline{k}^{2}\right)$ takes the value $M(0)=a+c=0.778572 \mathrm{GeV}$. Figure 1 depicts our view (8) on $M\left(\underline{k}^{2}\right)$ for both double-logarithmic and linear scales.

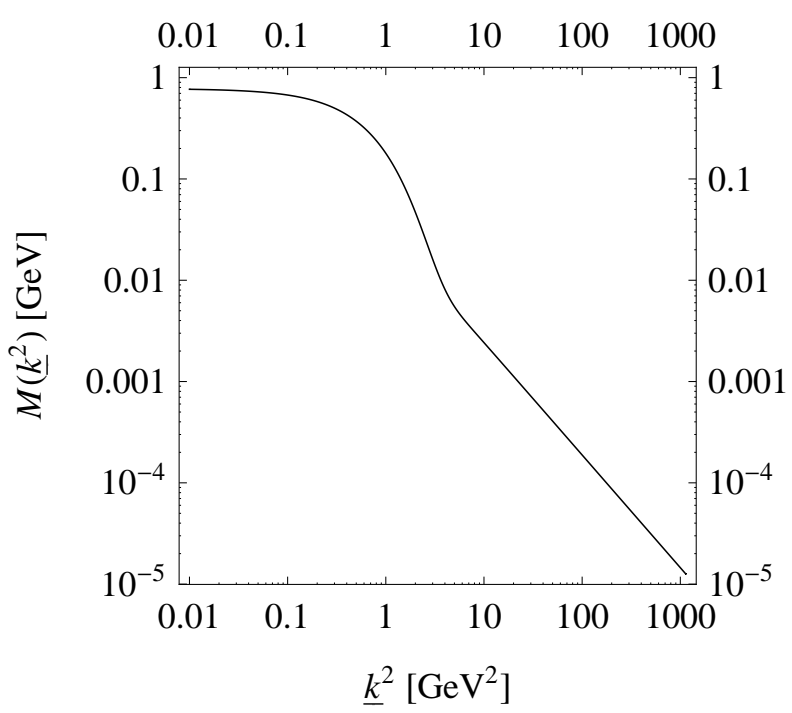

(a)

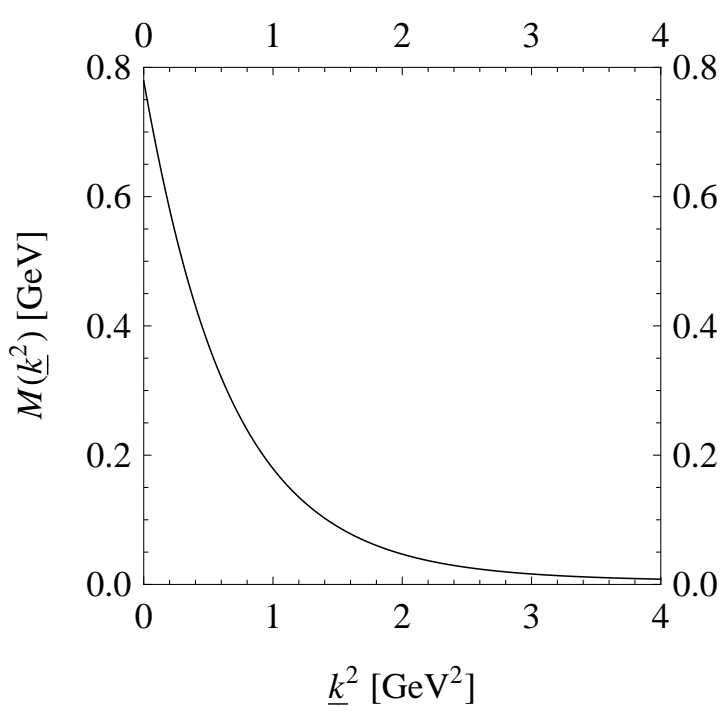

(b)

Figure 1: Mass function $M\left(\underline{k}^{2}\right)$, derived by solution of the renormalization-group-improved rainbow-ladder approximation to the Dyson-Schwinger equation for the quark propagator, $S(\underline{k})$, in the chiral limit (Fig. 2 of Ref. [13]), for double-logarithmic (a) and linear (b) scales.

2. Inserting $M\left(\underline{k}^{2}\right)$ in form of its parametrization (8) into our starting-point relation (7) takes us to the Goldstone-boson Bethe-Salpeter amplitude $\Phi(\underline{k}, 0)$. Mimicking the $p_{0}$ integration requested by the definition (1) by an integration of $\Phi(\underline{k}, 0)$ with respect to $\underline{k}_{4}$ yields the Salpeter amplitude $\phi(\boldsymbol{p})$. For the outcome of this numerical integration, the function $\varphi_{2}(\boldsymbol{p})$ multiplying the Dirac matrix $\gamma_{5}$, a nearly perfect fit may be found,

$$
\varphi_{2}(p)=\frac{a}{\left(b^{2}+p^{2}+c p^{4}\right)^{\gamma}}, \quad\left\|\varphi_{2}\right\|^{2} \equiv \int_{0}^{\infty} \mathrm{d} p p^{2}\left|\varphi_{2}(p)\right|^{2}=1,
$$


Table 2: Numerical values of the four parameters $(a, b, c, \gamma)$ controlling the behaviour (9) of the (sole) component $\varphi_{2}(p)$ of the Salpeter amplitude (3) of a spin-singlet quark-antiquark bound state fixed by insertion of our parametrization (8) of the quark mass function $M\left(\underline{k}^{2}\right)$ into the "starting-point" relationship (7) and subsequent integration (1) with respect to $\underline{k}_{4}$.

\begin{tabular}{lrrrr}
\hline \hline Parameter & $a\left[\mathrm{GeV}^{2 \gamma-\frac{3}{2}}\right]$ & $b[\mathrm{GeV}]$ & $c\left[\mathrm{GeV}^{-2}\right]$ & $\gamma$ \\
\hline Value & 8.7344 & 1.23635 & 2.57541 & 1.77044 \\
\hline \hline
\end{tabular}

with the numerical values of its just four parameters $a, b, c$, and $\gamma$ revealed in Table 2 . Figure 2 confronts, for the Salpeter amplitude $\varphi_{2}(p)$, its parametrization (9) with the direct output of the integration (1). The maximum error is less than $0.0036 \mathrm{GeV}^{-3 / 2}$.

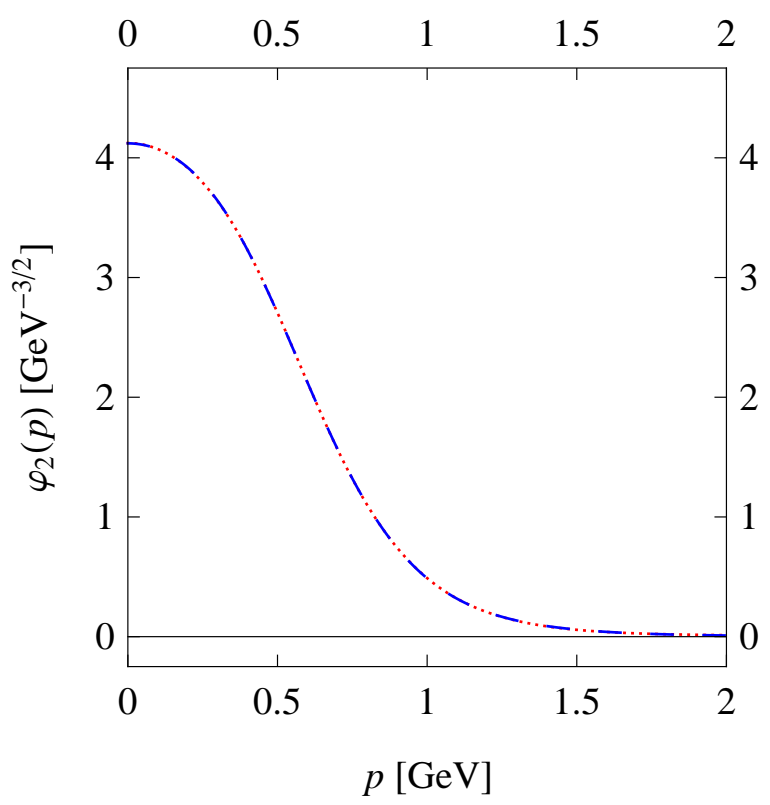

Figure 2: Comparison of the result of a numerical integration, according to definition (1), of the Salpeter amplitude $\Phi(\underline{k}, 0)$ emerging from the quark mass function (8) (red dotted line) with our parametrization (9) defined by the parameter values of Table 2 (blue dashed line).

3. Clearly, for the envisaged extraction of any underlying interaction potential $V(r)$, we next have to move to configuration space. It goes without saying that, in the course of performing the required Fourier transformations, we would like to maintain sufficient control about the actual reliability of our inversion technique. We may feel entitled to claim to have achieved such goal if we are able to estimate the accuracy of our results. To this end, we would like to perform the remaining intermediate steps of the present inversion approach by as far as reasonable analytical means. Consequently, in spite of having at our disposal, in form of Eq. (9), a parametrization pretty close to optimum, we prefer to proceed by use of an approximation which is of slightly minor quality but 
resembles the single-particle kinetic energy $E(p)$ sufficiently to provide, for particular values of the quark mass, analytic access to $V(r)$. Hence, we continue with the ansatz

$$
\varphi_{2}(p) \approx \sqrt{\frac{\Gamma(2 \gamma)}{\sqrt{\pi} \Gamma\left(2 \gamma-\frac{3}{2}\right)}} \frac{2 b^{2 \gamma-\frac{3}{2}}}{\left(p^{2}+b^{2}\right)^{\gamma}}, \quad\left\|\varphi_{2}\right\|^{2} \equiv \int_{0}^{\infty} \mathrm{d} p p^{2}\left|\varphi_{2}(p)\right|^{2}=1,
$$

for $\gamma>\frac{3}{4}$, with the appropriate values of the two parameters $b$ and $\gamma$ given in Table 3 .

Table 3: Numerical values of the (just two) parameters $b, \gamma$ determining our "user-friendly" approximate parametrization (10) of the single component $\varphi_{2}(p)$ of the Salpeter amplitude (3) of spin-singlet quark-antiquark bound states derived from the quark mass function (8).

\begin{tabular}{lcc}
\hline \hline Parameter & $b[\mathrm{GeV}]$ & $\gamma$ \\
\hline Value & 1.69334 & 6.49292 \\
\hline \hline
\end{tabular}

The proximity of convenient fit (10) to "perfect" fit (9) may be judged from Fig. 3(a).

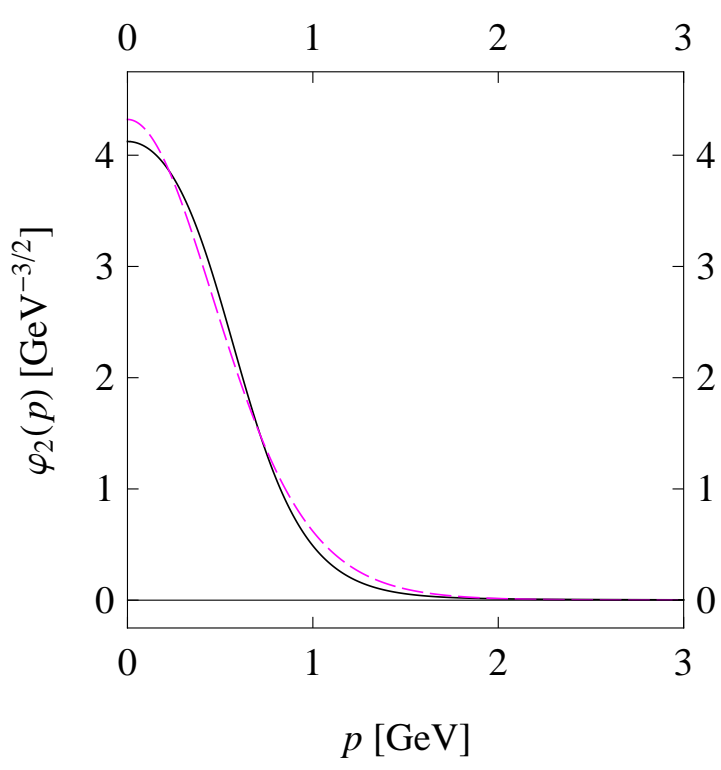

(a)

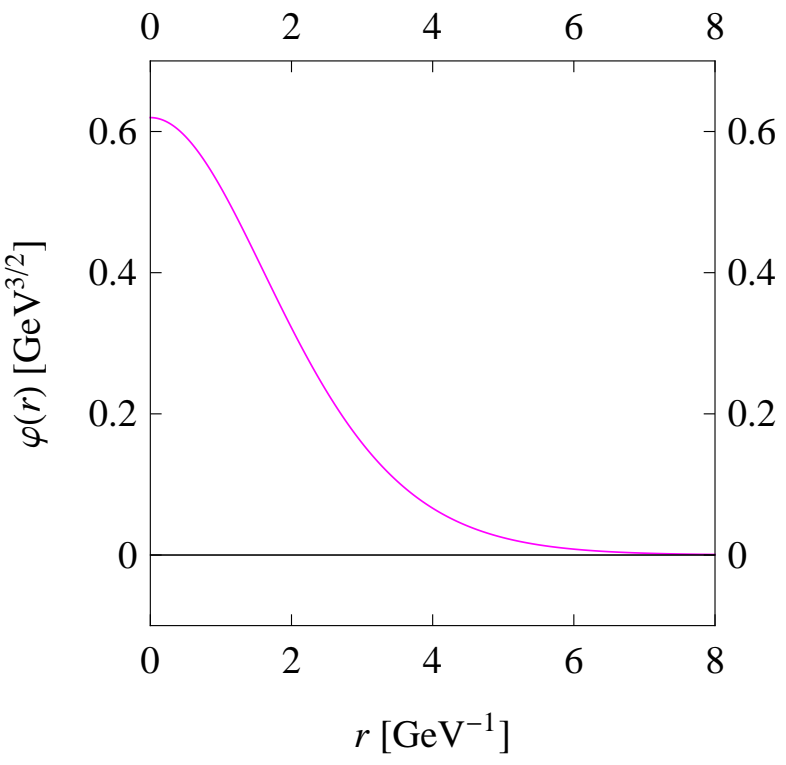

(b)

Figure 3: Normalized radial independent Salpeter component defining, at least for Salpeter equations (2) characterized by the Lorentz structure $2 \Gamma \otimes \Gamma=\gamma_{\mu} \otimes \gamma^{\mu}+\gamma_{5} \otimes \gamma_{5}-1 \otimes 1$ of their interaction kernels, the Salpeter amplitude (3) of any spin-singlet state, thus, in particular, of any pseudoscalar meson. In momentum-space representation (a) we show its exact shape computed, via the relationship (7), from the quark mass function of Fig. 1 (black solid line) as well as an approximation deduced from a fit to the simplified functional dependence (10) (magenta dashed line) whereas in configuration-space representation (b) we only depict the behaviour of the Fourier-Bessel transform of the latter approximation (magenta solid line). 
For the marginally simpler parametrization (10), it is then straightforward to obtain, by Fourier-Bessel transformation of $\varphi_{2}(p)$, the Salpeter component $\varphi(r)$ analytically, in terms of the modified Bessel functions $K_{\eta}(z)$ of the second kind of order $\eta \in \mathbb{R}[14]$ :

$\varphi(r)=\sqrt{\frac{\Gamma(2 \gamma)}{\sqrt{\pi} \Gamma\left(2 \gamma-\frac{3}{2}\right)}} \frac{2^{2-\gamma} b^{\gamma}}{\Gamma(\gamma)} r^{\gamma-\frac{3}{2}} K_{\frac{3}{2}-\gamma}(b r), \quad\|\varphi\|^{2} \equiv \int_{0}^{\infty} \mathrm{d} r r^{2}|\varphi(r)|^{2}=1$.

Figure 3(b) illustrates this configuration-space behaviour of our Salpeter component.

4. In general, the kinetic term's Fourier-Bessel transform $T(r)$ can be computed merely by a numerical integration. Thereafter, according to Eq. (6), this inversion procedure is easily completed by computing the sought potential $V(r)$ by dividing $T(r)$ by $\varphi(r)$. The behaviour of the resulting potentials for various quark masses is shown in Fig. 4.

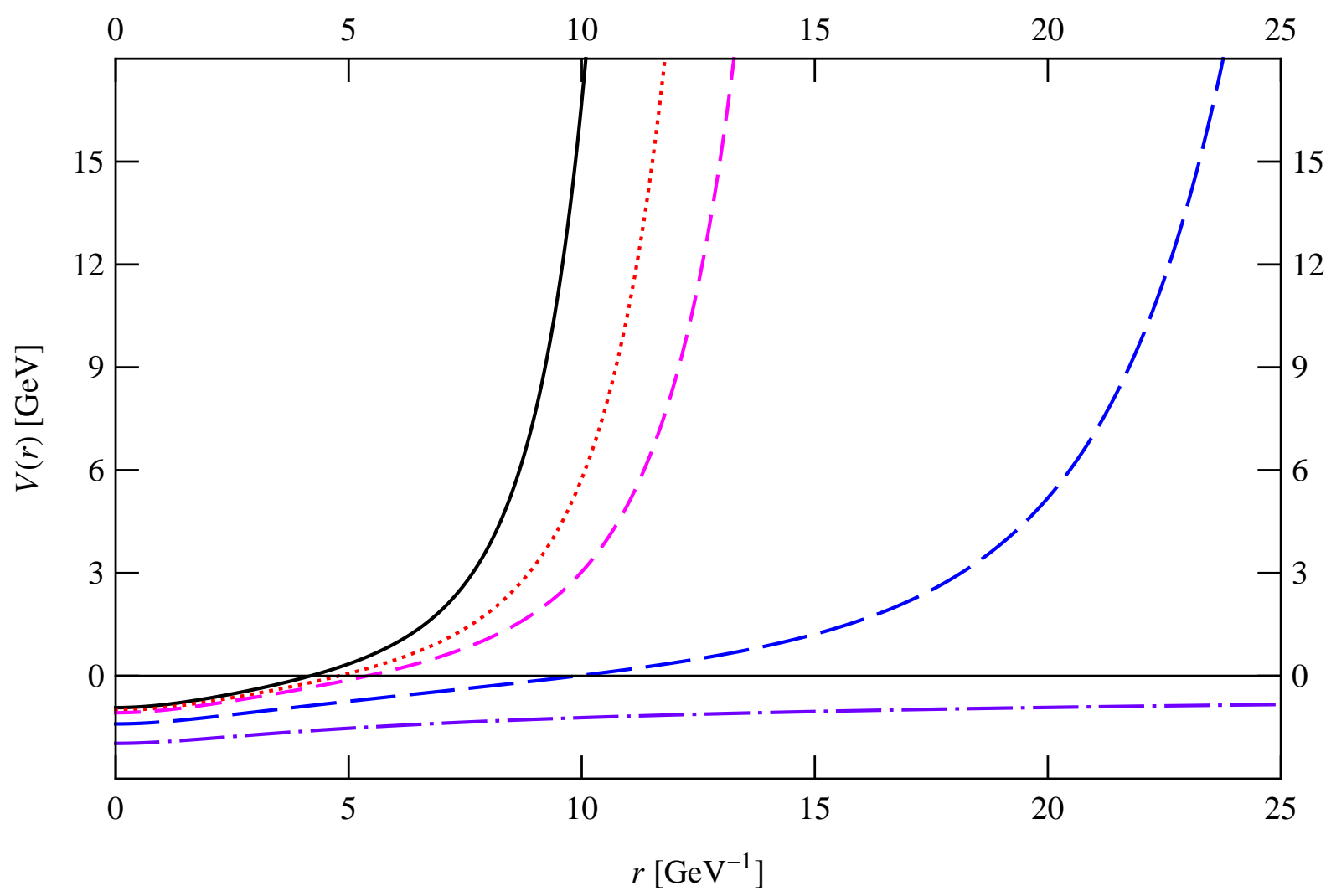

Figure 4: Configuration-space potential $V(r)$ extracted from the Salpeter equation (2) with interaction-kernel Lorentz structure $2 \Gamma \otimes \Gamma=\gamma_{\mu} \otimes \gamma^{\mu}+\gamma_{5} \otimes \gamma_{5}-1 \otimes 1$, for typical quark-mass value, $m: m=0$ (black solid line), $m=0.35 \mathrm{GeV}$ (red dotted line), $m=0.5 \mathrm{GeV}$ (magenta short-dashed line), $m=1 \mathrm{GeV}$ (blue long-dashed line), and $m=b$ (violet dot-dashed line).

As promised when motivating our step 3, owing to the pivotal simplification (10), for, at least, two distinct choices of the quark mass $m$ the Fourier-Bessel transform of the kinetic term $E(p) \varphi_{2}(p)$, and thus the potential $V(r)$, can be found in analytical form:

(a) If that common quark mass $m$ vanishes, that is, for $m=0$, the kinetic term $T(r)$ may be formulated by use of the modified Bessel functions of the first kind $I_{\eta}(z)$, of order $\eta \in \mathbb{R}[14]$, and the modified Struve functions $\mathbf{L}_{\eta}(z)$, of order $\eta \in \mathbb{R}[14]$ : 


$$
\begin{aligned}
T(r) & =\sqrt{\frac{\pi^{\frac{3}{2}} \Gamma(2 \gamma)}{\Gamma\left(2 \gamma-\frac{3}{2}\right)}} \frac{2^{1-\gamma}}{\sin (\gamma \pi) \Gamma(\gamma)} \frac{1}{r^{\frac{5}{2}}} \\
& \times\left[2(1-\gamma)(b r)^{\gamma} I_{\gamma-\frac{3}{2}}(b r)-(b r)^{1+\gamma} I_{\gamma-\frac{1}{2}}(b r)\right. \\
& \left.+(b r)^{\gamma} \mathbf{L}_{\frac{3}{2}-\gamma}(b r)+(b r)^{1+\gamma} \mathbf{L}_{\frac{5}{2}-\gamma}(b r)+\frac{\sin (\gamma \pi)}{2^{\frac{3}{2}-\gamma} \pi^{\frac{3}{2}}} \Gamma(\gamma-2)(b r)^{\frac{5}{2}}\right] .
\end{aligned}
$$

We do not see a genuine need for reproducing here the rather lengthy expression of $V(r)$ that arises upon division of the above $T(r)$ by $\varphi(r)$ : see the solid curve in Fig. 4. With this explicit expression for $V(r)$ at hand, it is trivial to characterize the behaviour of $V(r)$ in the limit of either small or large interquark distances $r$ :

$$
\begin{aligned}
& V(0)=-\frac{2 b \sqrt{\pi}}{\sin (\gamma \pi) \Gamma(3-\gamma) \Gamma\left(\gamma-\frac{3}{2}\right)} \quad \text { for } \gamma>2, \\
& V(r) \underset{r \rightarrow \infty}{\longrightarrow} \frac{\gamma(\gamma-1)}{4 \sin (\gamma \pi)} \frac{\exp (2 b r)}{r} .
\end{aligned}
$$

Thus, for appropriate values of $\gamma$, we get a confinement-betraying rise to infinity:

$$
V(r) \underset{r \rightarrow \infty}{\longrightarrow}+\infty \quad \text { for } \gamma \in(2 n, 2 n+1), \quad n=1,2,3, \ldots .
$$

By formula (6.1.17) of Ref. [14], the expression $\sin (\gamma \pi) \Gamma(3-\gamma)$ is nonvanishing,

$$
\sin (\gamma \pi) \Gamma(3-\gamma)=\frac{\pi}{\Gamma(\gamma-2)}>0 \quad \text { for } \gamma>2 ;
$$

accordingly, at spatial origin $r=0$, the potential $V(r)$ assumes a finite value: for the parameters of Table 3, $V(0)=-0.926813 \mathrm{GeV}$ (cf. the solid curve in Fig. 4).

(b) If, by chance, the common quark mass $m$ is exactly equal to the mass parameter $b$ in our ansatz (10), that is, for $m=b$, the kinetic term $T(r)$ turns out to involve only the modified Bessel functions of the second kind $K_{\eta}(z)$, of order $\eta \in \mathbb{R}[14]$ :

$$
T(r)=\sqrt{\frac{\Gamma(2 \gamma)}{\sqrt{\pi} \Gamma\left(2 \gamma-\frac{3}{2}\right)}} \frac{2^{\frac{5}{2}-\gamma} b^{\frac{1}{2}+\gamma}}{\Gamma\left(\gamma-\frac{1}{2}\right)} r^{\gamma-2} K_{2-\gamma}(b r) .
$$

With the overall normalization dropping out, the emerging potential $V(r)$ reads

$$
V(r)=-\frac{\Gamma(\gamma)}{\Gamma\left(\gamma-\frac{1}{2}\right)} \sqrt{\frac{2 b}{r}} \frac{K_{2-\gamma}(b r)}{K_{\frac{3}{2}-\gamma}(b r)} .
$$

This behaviour of $V(r)$ is reflected graphically by the dot-dashed curve in Fig. 4. For such simple $V(r)$ shape, its $r \rightarrow 0$ and $r \rightarrow \infty$ limits can be directly read off:

$$
\begin{aligned}
& V(0)=-\frac{b \Gamma(\gamma-2) \Gamma(\gamma)}{\Gamma\left(\gamma-\frac{3}{2}\right) \Gamma\left(\gamma-\frac{1}{2}\right)} \quad \text { for } \gamma>2, \\
& V(r) \underset{r \rightarrow \infty}{\longrightarrow}-\frac{\Gamma(\gamma)}{\Gamma\left(\gamma-\frac{1}{2}\right)} \sqrt{\frac{2 b}{r}} \underset{r \rightarrow \infty}{\longrightarrow} 0 .
\end{aligned}
$$

Thus, for rising separation $r$, this potential starts from a finite, negative value at the origin $r=0$, namely, $V(0)=-1.96928 \mathrm{GeV}$ for the two parameter values of Table 3, but stays below zero even for $r \rightarrow \infty$; cf. the dot-dashed curve in Fig. 4 .

In general, the Fourier-Bessel transform of the kinetic term, $T(r)$, and, consequently, the resulting potential, $V(r)$, have to be computed by numerical integration. Figure 4 
presents the outcomes of such undertaking, for a couple of selected quark masses $m$ in the interval $0 \leq m \leq b$. Needless to say that, in both analytically accessible cases, our numerical results exhibit perfect agreement with the above explicit findings for $V(r)$.

Numerically, the oscillatory behaviour induced by the very definition of the Fourier-Bessel transformation requires in its application a particularly careful analysis. In view of this, the possibility of the independent verification of one's numerical findings, for exceptional cases, by corresponding analytic results should be considered as a precious and welcome bonanza.

\section{Summary, Conclusion, Interpretation and Outlook}

Abandoning for the moment most of our previous ambitions towards analytic treatments of the lightest pseudoscalar mesons within the formalism of the instantaneous Bethe-Salpeter approach [4-6], in the present analysis we derived, for the Salpeter equation (2), the form of that interaction kernel which is capable of describing Goldstone-type pseudoscalar mesons, from the quark mass function, rendered accessible by a fundamental relationship (resulting from the chiral symmetry of QCD $[12,13]$ ), between the quark propagator, on the one hand, and the meson Bethe-Salpeter amplitude, on the other hand. For increasing effective quark mass $m$, the gross behaviour of $V(r)$ resembles that observed in Ref. [5]: a rise to infinity for sufficiently small $m$ but an approach to a finite nonpositive value for larger $m$. This is in full accordance with the inevitable large- $m$ behaviour of $V(r)$ already demonstrated in Ref. [5]:

$$
E(p) \underset{m \rightarrow \infty}{\longrightarrow} m \quad \Longrightarrow \quad T(r) \underset{m \rightarrow \infty}{\longrightarrow} m \varphi(r) \quad \Longrightarrow \quad V(r) \underset{m \rightarrow \infty}{\longrightarrow}-m .
$$

The origin of any such qualitatively different behaviour of the derived potentials $V(r)$ is easily identified: For given mass $m$ of the bound-state constituents, the configuration-space quantities $T(r)$ and $\varphi(r)$, as mere Fourier-Bessel transforms, and thus likewise their ratios, the potentials $V(r)=-T(r) / \varphi(r)$, are unambiguously determined already by the Salpeter component $\varphi_{2}(p)$ forming the exclusive momentum-space input of our inversion procedure. Thus, any substantial differences of the predictions for $V(r)$ have to be attributed to $\varphi_{2}(p)$. For the sake of comparison, let us represent the approximate behaviour of $\varphi_{2}(p)$ in the form

$$
\varphi_{2}(p) \propto \frac{1}{\left(p^{2}+\mu^{2}\right)^{\nu}}
$$

with some characteristic mass scale, $\mu$, and discuss the associated exponents $\nu$ encountered in this and two previous analyses: In Ref. [5], the momentum dependence of the chiral-limit quark mass function in the ultraviolet limit of large spacelike momenta, deduced on general grounds [13], implied $\nu=\frac{3}{2}$. In Ref. [6], we modelled the (confinement-promoting) presence of an inflection point in the quark mass function by an admixture that entailed a somewhat modified value of the effective $\nu$, depending on the relative amount of this admixture. Here, this exponent is, of course, equal to the numerical value of the parameter $\gamma$ in the simplified parametrization (10), $\nu=\gamma \approx \frac{13}{2}$ according to Table 3 , and markedly larger, because in the simplified form (10) this parameter must take care of the $p^{4}$ term in the parametrization (9) which, otherwise, would dominate the behaviour of $\varphi_{2}(p)$ at large $p$. This is reflected by the nonsingular behaviour of $\varphi(r)$ at the origin, whence there is no need for a counterbalancing singularity of $V(r)$ at the origin that allows for the sought masslessness of the bound states.

So far, we have harvested our (analytical or numerical) input in Euclidean space, i.e., at spacelike quark momenta. Beyond doubt, it will be interesting to move to Minkowski space and to exploit findings for timelike quark momenta, to the extent such results are available. 


\section{References}

[1] H. A. Bethe and E. E. Salpeter, Phys. Rev. 82 (1951) 309;

M. Gell-Mann and F. Low, Phys. Rev. 84 (1951) 350;

E. E. Salpeter and H. A. Bethe, Phys. Rev. 84 (1951) 1232.

[2] W. Lucha and F. F. Schöberl, J. Phys. G: Nucl. Part. Phys. 31 (2005) 1133, arXiv:hepth/0507281.

[3] E. E. Salpeter, Phys. Rev. 87 (1952) 328.

[4] W. Lucha and F. F. Schöberl, Phys. Rev. D 87 (2013) 016009, arXiv:1211.4716 [hep-ph].

[5] W. Lucha, Proc. Sci., EPS-HEP 2013 (2013) 007, arXiv:1308.3130 [hep-ph];

W. Lucha and F. F. Schöberl, Phys. Rev. D 92 (2015) 076005, arXiv:1508.02951 [hep-ph].

[6] W. Lucha and F. F. Schöberl, Phys. Rev. D 93 (2016) 056006, arXiv:1602.02356 [hep-ph].

[7] W. Lucha and F. F. Schöberl, in QCD@Work 200\%: International Workshop on Quantum Chromodynamics: Theory and Experiment, edited by P. Colangelo, D. Creanza, F. De Fazio, R. A. Fini, E. Nappi, and G. Nardulli, AIP Conf. Proc. No. 964 (AIP, New York, 2007), p. 318, arXiv:0707.1440 [hep-ph].

[8] Z.-F. Li, W. Lucha, and F. F. Schöberl, Phys. Rev. D 76 (2007) 125028, arXiv:0707.3202 [hep-ph].

[9] Z.-F. Li, W. Lucha, and F. F. Schöberl, J. Phys. G: Nucl. Part. Phys. 35 (2008) 115002, arXiv:0712.2947 [hep-ph].

[10] J.-F. Lagaë, Phys. Rev. D 45 (1992) 305;

M. G. Olsson, S. Veseli, and K. Williams, Phys. Rev. D 52 (1995) 5141, arXiv:hep$\mathrm{ph} / 9503477$.

[11] W. Lucha and F. F. Schöberl, Int. J. Mod. Phys. A 07 (1992) 6431; in Proceedings of the International Conference on Quark Confinement and the Hadron Spectrum, edited by N. Brambilla and G. M. Prosperi (World Scientific, River Edge, NJ, 1995) p. 100, arXiv:hepph/9410221; Int. J. Mod. Phys. A 14 (1999) 2309, arXiv:hep-ph/9812368; Fizika B 8 (1999) 193, arXiv:hep-ph/9812526; Recent Res. Devel. Phys. 5 (2004) 1423, arXiv:hep-ph/0408184.

[12] P. Maris, C. D. Roberts, and P. C. Tandy, Phys. Lett. B 420 (1998) 267, arXiv:nuclth/9707003.

[13] P. Maris and C. D. Roberts, Phys. Rev. C 56 (1997) 3369, arXiv:nucl-th/9708029.

[14] Handbook of Mathematical Functions, edited by M. Abramowitz and I. A. Stegun (Dover, New York, 1964). 